\title{
Nonequilibrium roughening transition in a simple model of fungal growth in $1+1$ dimensions
}

\author{
Juan M. López and Henrik Jeldtoft Jensen \\ Department of Mathematics, Imperial College, 180 Queen's Gate, London SW7 2BZ, United Kingdom
}

\begin{abstract}
We introduce a simple model of yeast-like growth of fungi colonies, which exhibits a continuous roughening transition far from equilibrium from a smooth $(\alpha=0)$ to rough phase $(\alpha=1 / 2)$ in $1+1$ dimensions. At the transition some scaling exponents are calculated by mapping the problem onto a directed percolation process. The model reproduces the roughening transition observed in some experiments of fungal growth.
\end{abstract}

PACS numbers:87.10.+e,05.40.+j,61.43.-j,68.35.Rh

Much effort has been devoted to the search for the basic principles governing the pattern formation in living organisms. Among all the phenomena of formation and development of complex structures involving living organisms, the growth of bacteria and fungi colonies has attracted a considerable amount of work in recent years [1] 12.

Besides the formation of patterns, which is associated with the existence of some unstable modes, spatiotemporal scale invariance is also commonly observed depending on the environmental conditions [7 10]. In the particular case of fungi colonies, the morphology may be well classified in hyphal and yeast-like growth [13]. The former corresponds to multicellular growth and fractal filamentous patterns form due to the existence of highly cooperative behavior of the individual cells. However, yeast-like growth occurs in solidified media and in this case the colony is a very compact object. The front of the colony usually becomes rough (i.e. the interface width diverges with the linear size of the system) and its dynamics is completely characterized by the critical exponents, which describe the scaling properties of the interface fluctuations 14.

In recent experiments [12] with the yeast Pichia membranaefaciens on solidified agarose film, several morphological transitions in $1+1$ dimensions have been reported. In these experiments different front morphologies were obtained depending on the concentration of polluting metabolites. Also transitions from rough to flat interfaces were observed [15], although never studied systematicly. In the case of Bacillus subtilis, the existence of completely smooth and Eden-like morphologies (among others) depends on the agar hardness and nutrients concentration 11. Our attention in this Letter is focused on this roughening transition.

In $1+1$ dimensions a surface under equilibrium conditions is rough at any finite temperature, because thermal fluctuations always make the flat surface entropically unfavorable. Only in higher dimensions can equilibrium interfaces undergo a phase transition from a rough to a smooth interface at some critical temperature. The situation in nonequilibrium is much richer because detailed balance is not required and growth processes may exhibit a roughening transition even in $1+1$ dimensions, although examples are few [16 18]. More generally, phase transitions in nonequilibrium $1+1$ dimensional systems have usually been observed in systems with absorbing states. Thus, it is of great interest to find models far from equilibrium which do not possess absorbing states but still display a phase transition.

In this Letter we introduce a new class of models directly inspired by yeast-like fungal growth in $1+1$ dimensions. The model has no absorbing states and exhibits a nonequilibrium roughening transition between a flat and a rough phase. Some scaling properties of the model at the transition can be related to directed percolation. We obtain that close to the transition a diverging correlation length $\xi \sim|\epsilon|^{-\nu}$ appears, where $\epsilon$ is the distance to the critical point, and the critical exponent is in good agreement with directed percolation in $1+1$ dimensions, $\nu \approx \nu_{\perp} \approx 1.10$. We find that, in the rough phase, the model belongs to the Edwards-Wilkinson [19 universality class.

Model.- We study a class of models motivated by the growth of colonial organisms like fungi and bacteria. The model is defined on a $1+1$ dimensional lattice in which every site can have two different states: occupied or vacant. Growth of the colony occurs because of the division of individual cells, so only nearest neighbors of occupied sites can potentially become occupied. The basic idea now is that cell division is less likely in young cells, so the probability for a vacant site $i$ of being occupied in the next time step has to increase with the total age $A_{i}(t)$ of the occupied nearest neighboring sites of that vacant site $i$. At every time step a growth probability

$$
P_{i}(t)=F\left(\theta \eta_{i} A_{i}(t)\right)
$$

is assigned to all vacant sites that are nearest neighbors of occupied sites (note that $A_{i}=0$ at the remaining vacant sites). $F(x)$ is any monotonous increasing function that satisfies $F(0)=0$ and $F(\infty)=1$. For definiteness, in our simulations we simply used $F(x)=\tanh (x)$. $\theta$ is an external parameter which controls the growth rate. The variation in the aging times among the individual 
cells in the colony is modelled by the parameter $\eta_{i}$. This random variable is quenched, uncorrelated and uniformly distributed in $[1-\Delta, 1+\Delta]$. The simulations are performed on a triangular lattice with open boundary conditions, parallelly updating all the sites according to the growth probability (1D). The model produces a very compact bulk and irrelevant overhangs at the scale of the lattice spacing may appear on the growth front depending on the tunning parameter $\theta$.

From the point of view of the biological process, this model has to be considered as a simplified version of a more realistic description. The experiments mentioned above [12] have shown that yeast-like growth of fungi strongly depends on the concentration of nutrients and inhibitors in the medium. In a more complicated model [20] the effect of inhibitors can be studied by including an inhibitors diffusive field $c_{i}(t)$ which is coupled with the growth probability at every site. The probability for a vacant site $i$ of becoming occupied is then given by $P_{i}(t)=F\left(\theta \eta_{i} A_{i}(t) \exp \left(-c_{i}(t) / c_{0}\right)\right)$, where $c_{0}$ is a threshold concentration above which the effect of inhibitors begins to be relevant. The model we are presenting here corresponds to the limit $c_{0} \rightarrow \infty$.

Continuous Roughening Transition.- Despite its simplicity the model has a non trivial behavior. For small values of the parameter $\theta$, the fungus front generated by the model becomes rough and its dynamics is characterized as usual by two kinetic roughening exponents [14]. In contrast, for large values of $\theta$, the interface becomes flat even when started from a non smooth initial condition. This phase transition occurs for any value of the disorder intensity $\Delta$. We found that these two phases are separated by a critical threshold which depends on the degree of disorder and is at $\theta_{c}=0.183 \pm 0.003$ for the case of no disorder $\Delta=0$. In the following we restrict ourselves to the homogeneous case $\Delta=0$ although similar results are found for any $0 \leq \Delta<1$.

Let us first study the rough phase, $\theta \ll \theta_{c}$, in some detail. Denoting by $h(x, t)$ the front height at time $t$ and substrate position $x$, the interface width in a system of size $\left.L, W(L, t)=\overline{\left\langle[h(x, t)-\bar{h}(t)]^{2}\right.}\right\rangle^{1 / 2}$ (where overbar and $\langle\cdots\rangle$ denote spatial average and over realizations respectively), measures the magnitude of the interface fluctuations. The width is expected to satisfy the dynamic scaling ansatz 21],14]:

$$
W(L, t) \sim \begin{cases}t^{\beta} & \text { if } t \ll L^{z} \\ L^{\alpha} & \text { if } t \gg L^{z}\end{cases}
$$

where $\alpha$ is the roughness exponent and $z=\alpha / \beta$.

We have performed simulations in systems of different sizes to determine the universality class of the model in the rough phase. Figure 1 shows that sufficiently far from the transition $(\theta=0.01)$ the scaling behavior of the interface is given by Eq.(2). The kinetic roughening exponents $\alpha=0.46 \pm 0.05$ and $\beta=0.24 \pm 0.02$ indicate that the model belongs to the Edwards-Wilkinson universality class 19].

Approaching the roughening transition from the rough phase a new diverging length $\xi \sim \epsilon^{-\nu}$, where $\epsilon=\theta_{c}-\theta$, appears and enters the scaling of the width. Simulations close to the transition $\left(\epsilon \rightarrow 0^{+}\right)$were performed in systems of different sizes. In Fig. 2 results for $W(L, t)$ vs. time corresponding to $L=900$ and average over 50 realizations are plotted. We found that there exist two different regimes separated by a crossover time $t_{c} \sim \epsilon^{-\gamma}$, which diverges as $\epsilon \rightarrow 0^{+}$. Figure 2 shows that, near and below the transition, $W(L, t)$ scales as $\epsilon^{-\kappa_{F}}$ for times $t \ll t_{c} \ll L^{z}$ and $W(L, t) \sim t^{\beta} \epsilon^{\kappa_{R}}$ for $t_{c} \ll t \ll L^{z} . \kappa_{F}\left(\kappa_{R}\right)$ stands for the scaling exponent in the flat (rough) regime of the surface width as the transition is approached. For times $t \gg L^{z}$ the interface gets to saturation as in (2).

The scaling behavior in the intermediate time regime before saturation suggests the following scaling ansatz for times $t \ll L^{z}$ :

$$
W(L, t, \epsilon)=\epsilon^{-\kappa_{F}} g\left(t / t_{c}\right),
$$

where the scaling function is $g(u) \sim$ const if $u \ll 1$ and $g(u) \sim u^{\beta}$ for $u \gg 1$. The scaling relation

$$
\kappa_{R}+\kappa_{F}=\beta \gamma
$$

between critical exponents must also be fulfilled. The inset of Fig. 2 shows the data collapse for the exponents $\gamma=1.73 \pm 0.03$ and $\kappa_{F}=0.02$ is consistent with Eq.(3) over a range of more than four decades. Due to the very small value of $\kappa_{F}$ a logaritmic dependence $W \sim \log (\epsilon)$ cannot be ruled out. Using the scaling relation (何) we find $\kappa_{R} \simeq 0.41$, which is to be compared with the the measured value $\kappa_{R} \simeq 0.42 \pm 0.01$.

As seen in some other $1+1$ dimensional growth models exhibiting a roughening transition [17,18, some scaling properties are related to directed percolation (DP) [22. Our model turns out to be a model with a maximal velocity. Small values of $\theta$ produce small growth rates and the front propagates with a finite velocity. If the parameter $\theta$ is increased, the probability for a site of growing (i.e. becoming occupied) is also raised as follows from Eq.(11). A given site reaches the maximal velocity when its probability of growing at the next time step is one and then no further increase of the propagation velocity at that site can occur [23]. The transition from rough to flat surface is a DP transition. The flat phase corresponds to the active DP phase whereas the rough phase corresponds to the non-active DP phase. The sites at the highest level $h=v_{\max } t$ are active sites of a DP process. Below the transition (rough phase) there are no sites growing at maximal velocity and the surface mean velocity is less than $v_{\max }$. Above the transition (flat phase), on the contrary, there is a finite density of sites advancing at $v_{\max }$. At $\theta=\theta_{c}$ the sites which grow with maximum velocity 
constitute a percolation cluster directed along the growth direction, as depicted in Fig. 3.

The mapping to DP allows the derivation of several scaling exponents. The correlation length $\xi$ is identified with the correlation length of the DP clusters $\xi_{\perp}$, which is transversal to the growth direction of the interface, and $\nu=\nu_{\perp} \approx 1.10$ in $1+1$ dimensions. This implies that the crossover time $t_{c} \sim \xi^{z^{\prime}}$ in Eq.(3) is given by $t_{c} \sim \epsilon^{\nu_{\|}}$using the fact that $z^{\prime}=\nu_{\|} / \nu_{\perp}$ for DP. Our estimation of the crossover time exponent $\gamma=1.73 \pm 0.03$ (see Fig. 2) is in excellent agreement with the DP exponent $\nu_{\|} \approx 1.73$.

Order Parameters.- We now define a convenient order parameter for the rough phase $\left(\theta<\theta_{c}\right)$ as $V=v_{\max }-v$ with $v=\partial_{t} \bar{h}(t)$ the mean front velocity. The variable $V$ measures the fraction of the surface points propagating at velocity lower than the maximal one. $V$ is different from zero only in the rough phase and its scaling behavior can also be related to properties of the associated $\mathrm{DP}$ process. In the rough phase $V$ is given by the inverse of the typical time that the DP correlations survive $\tau \sim \xi_{\|}$, which is the only characteristic time scale. So, $V \sim 1 / \tau \sim \epsilon^{\nu \|}$ close to the transition. In our simulations we measured $V \sim \epsilon^{1.746 \pm 0.018}$, as shown in Fig. 4, in excellent agreement with the simple DP scaling picture.

As for the flat phase $\left(\theta>\theta_{c}\right)$ the density of sites at the maximal height is an appropriate order parameter [17, which corresponds to the density of active sites in DP. However, more interesting here may be the use of appropriate order parameters which take into account the symmetries of the model in order to study the possibility of spontaneous symmetry breaking (SSB) in the flat phase. It has been shown that SSB may take place in nonequilibrium situations under certain conditions even in $1+1$ dimensions 18,24.

The dynamical rules of our model are invariant under translation of an integer number of levels in the growth direction. The dynamics do not favor odd nor even heights. Thus it is adequate to define a magnetisationlike order parameter

$$
M(t)=\frac{1}{L} \sum_{i=1}^{L}(-1)^{h(i, t)},
$$

which is no conserved by the dynamics of the model. This order parameter can be used to quantify the symmetry breaking which may take place in the flat phase [18].

In our simulations we found that in the rough phase $\langle M\rangle=0$ and also $\langle|M|\rangle=0$, where $\langle\cdots\rangle$ denotes an average over realizations. In the rough phase the interface explores many height levels and the contribution to the magnetisation at different sites average out. On the contrary in the flat phase $\langle M\rangle=0$ but $\langle|M|\rangle \neq 0$ due to the fact that most heights are at the highest level. However, SSB was not observed in our model. For every realization $M(t)$ gets to a stationary state consisting of flips between $+\left|M_{s}\right|$ and $-\left|M_{s}\right|$ at almost every time step. In Ref. [18] a growth model with sequential updates has recently been studied in which SSB was found. In that model, SSB in the flat phase is related to the fact that the interface eventually becomes pinned at a certain height and the velocity is actually zero in the thermodynamic limit. Only in a finite system will the interface have a finite velocity which vanishes exponentially with the system size. This ensures the breaking of the symmetry between odd and even heights in the thermodynamic limit. The final interface will be pinned at an odd or even height, depending on the initial conditions, breaking the symmetry of the dynamics. On the contrary in our model the velocity $v_{\max }$ in the flat phase is always finite (even in the thermodynamic limit $L \rightarrow \infty$ ) not allowing SSB. As occurs in the model reported in Ref. 17] the smooth phase does not survive in our model under sequential dynamics, due to the random walk nature of sequential updates.

The behavior of the order parameter of the flat phase $\langle|M|\rangle$ near the transition gives a new scaling exponent $\eta$, which describes its decay as the transition is approached from above $\langle|M|\rangle \sim(-\epsilon)^{\eta}$. Our simulations indicate that $\eta \approx 0.50$ which can be compared with $\eta=0.55 \pm 0.05$ previously measured for a different model [18].

Conclusions.- We have studied a model for fungal growth in which the likelihood of growth at a given site depends on the local environment. Vacant sites surrounded by older cells are more likely to become occupied than sites neighboring younger cells. The model exhibits a roughening transition in $1+1$ dimensions as the growth rate is increased. We have found that the model belongs to the Edwards-Wilkinson universality in the rough phase. At the transition some critical exponents have been calculated by mapping the problem onto a DP process. Transition from a completely flat to rough interface has been observed by T. Sams et. al in experiments 15] with colonies of the yeast Pichia membranaefaciens on agarose film and by Wakita et. al. 11 in the bacterium Bacillus subtilis.

The authors thank A. Saeed for collaboration at the early stages of this work and M.J. Carlile, K. Christensen, R. Cuerno and T. Sams for useful comments. This work has been supported by the MEC of the Spanish Government and the European Commission.

[1] P.M. Iannaccone and M. Khokha Eds., Fractal Geometry in Biological Systems, CRS Press, Inc., Florida (1996);

[2] A.L. Cooper et. al., Proc. Roy. Soc. Lond. B 171, 175 (1968); ibid. 175, 95 (1970).

[3] E. Ben-Jacob, Contem. Phys. 38, 205 (1997).

[4] E. Ben-Jacob and P. Garik, Nature 343, 523 (1990) 
[5] E. Ben-jacob et. al., Nature 368, 46 (1994)

[6] D.A. Kessler and H. Levine, Phys. Rev. E 48, 4801 (1993).

[7] T. Vicsek et. al, Physica A 167, 315 (1990)

[8] M. Matsushita and H. Fujikawa, Physica A 16812294 (1990).

[9] S. Matsuura and S. Miyazima, Physica A 191, 30 (1992); and see also Fractals 1336 (1993).

[10] T. Matsuyama and M. Matsushita in Ref. [1].

[11] J. Wakita et. al., J. Phys. Soc. Jpn., 63, 1205 (1994).

[12] T. Sams et. al., Phys. Rev. Lett. 79, 313 (1997).

[13] M.J. Carlile and S.C. Watkinson, The Fungi, Academic Press, London (1994).

[14] A.-L. Barabási and H. E. Stanley, Fractal Concepts in Surface Growth (Cambridge University Press, Cambridge, (1995).

[15] Thomas Sams (Private Communication).

[16] R. Savit and R. Ziff, Phys. Rev. Lett. 55, 2515 (1985).

[17] J. Kertész and D.E. Wolf, Phys. Rev. Lett. 62, 2571 (1989).

[18] U. Alon et. al., Phys. Rev. Lett. 76, 2746 (1996).

[19] S. F. Edwards and D. R. Wilkinson, Proc. R. Soc. London A 381, 17 (1982).

[20] J.M. López and H.J. Jensen, to be published

[21] F. Family and T. Vicsek, J. Phys. A 18, L75 (1985).

[22] W. Kinzel, in Percolation Structures and Processes, edited by G. Deutscher et. al., Annals of the Israel Physical Society, Vol 5, Hilger, Bristol (1983).

[23] The maximal velocity in our model is given by one vertical lattice spacing per unit time.

[24] M.R. Evans et. al., Phys. Rev. Lett. 28, 208 (1995).

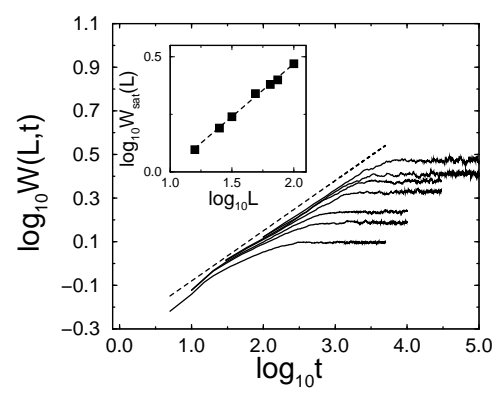

FIG. 1. Interface width of the front vs. time in the rough phase $(\theta=0.01)$ for different system sizes $L=16,25,32,50,64,75,100$. The slope of the dotted line corresponds to the time exponent $\beta=0.24 \pm 0.02$. In the inset the values of the width at saturation are plotted vs. system size. The dotted line fits the data and gives the roughness exponent $\alpha=0.46 \pm 0.05$.

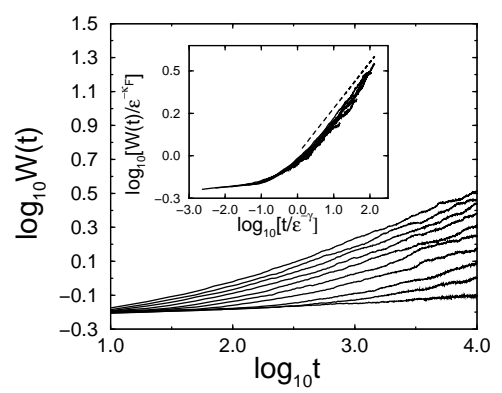

FIG. 2. Time behavior of the width in the rough phase near the transition. Curves correspond to different distances to the critical point, form $\epsilon=0.083$ (top) to $\epsilon=0.003$ (bottom). Data are collapsed in the inset following Eq.(3) for $\gamma=1.73$ and $\kappa_{F}=0.02$, where the dotted line has slope 0.25 .
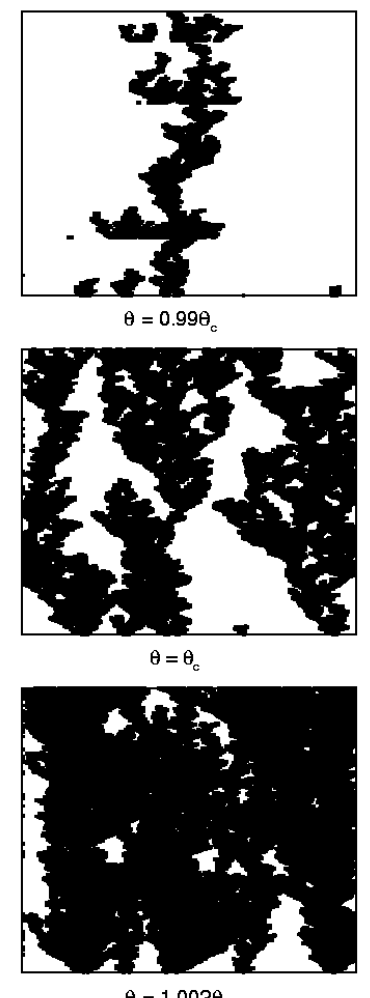

FIG. 3. Clusters of sites growing at maximal velocity. Every pixel represents the position $(x, h)$ of a site which moved at $v_{\max }$ and configurations at intervals of 5 time steps are shown. The system size is $L=500$ and time advances upwards.

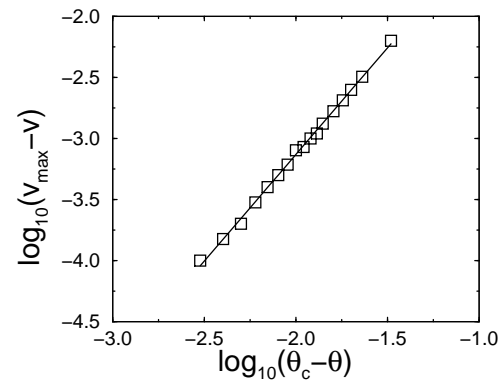

FIG. 4. Scaling of the order parameter $V$ in the rough phase near the transition. The line is a least-square fit of the data and has a slope $1.746 \pm 0.018$ in good agreement with $\nu_{\|}$. 